\title{
ANI scoring system - In differentiating alcoholic and non-alcoholic liver disease
}

\author{
Anitha $\mathbf{G}^{1}$, Sivakumar $\mathrm{J}^{2}$, Kalavathy Ponniraivan ${ }^{3}$ \\ ${ }^{1}$ Assistant Professor, Department of Biochemistry, Sri Venkateshwaraa Medical College and Hospital and Research \\ Centre, Puducherry, ${ }^{2}$ Associate Professor, Department of Biochemistry, Dhanalakshmi Srinivasan Medical College \\ and Hospital, Perambalur, ${ }^{3}$ Former Head and Professor, Department of Biochemistry, Trichy SRM Medical College and \\ Hospital, Trichy, Tamil Nadu, India
}

\section{A B S T R A C T}

Background: Alcoholic liver disease/non-alcoholic liver disease Index - ANI scoring system was constructed for clinically diagnosing the etiology of liver diseases, avoiding the risks of liver biopsy. Aims and Objectives: This study aims to test the reliability of ANI scoring system as a non-invasive method to distinguish alcoholic liver disease (ALD) from non-alcoholic fatty liver disease (NAFLD). Materials and Methods: This retrospective study was conducted on 177 (114 men and 58 women) treated as inpatients in Trichy SRM Medical College Hospital and Research Centre, Trichy, in the period of December 1, 2015-August 1, 2016. About 40 patients with etiology of viral, autoimmune, and biliary lithiasis were excluded. A total of 137 patients (98 men and 39 women) were classified into two groups, ALD (70) and NAFLD (67) based on diagnosis. Parameters of ANI - AST, ALT, MCV, BMI, and sex were recorded. ANI was calculated by online calculator. Results: ANI was significantly higher in patients with ALD than NAFLD $(P<0.01)$. The cutoff value of $A N I$ is -0.11 . Conclusion: On the basis of the results, ANI scoring system may be used in clinically distinguishing ALD from NAFLD, avoiding the risks of liver biopsy.

Key words: Alcoholic liver disease; Non-alcoholic fatty liver disease; ANI Score

Access this article online

Website:

http://nepjol.info/index.php/AJMS DOI: $10.3126 / a j m s . v 12 i 11.38868$

E-ISSN: 2091-0576

P-ISSN: 2467-9100

Copyright (c) 2021 Asian Journal of Medical Sciences

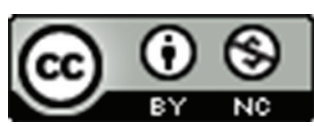

This work is licensed under a Creative Commons Attribution-NonCommercial 4.0 International License.

\section{INTRODUCTION}

Based on the etiology, fatty liver disease is classified into two broad categories, alcoholic liver disease (ALD) and non-alcoholic fatty liver disease (NAFLD). ${ }^{1}$ Both ALD and NAFLD impart serious health problems to people worldwide with prevalence raising to $32 \%$ among common Indian adult population ${ }^{2}$ and $70 \%$ among diabetic population. ${ }^{3}$ However, in practice, clinicians find it crucial to distinguish between alcoholic and non-alcoholic cause of hepatic steatosis, as the diagnosis of these two different entities directs to different selection of management. ${ }^{4,5}$

Although the gold standard to establish the diagnosis between ALD and NAFLD is liver biopsy, the procedure by itself is an invasive procedure and carries certain risks. ${ }^{6}$
Dunn et al., in the year 2006, formulated a new diagnostic model ALD/NAFLD index - ANI to differentiate alcoholic and non-ALD. ${ }^{7}$

This study aims to test the reliability of this ANI scoring system in distinguishing alcoholic and non-alcoholic liver disease.

\section{Aims and Objectives}

To test the reliability of this ANI scoring system in distinguishing Alcoholic and Nonalcoholic liver disease.

\section{MATERIALS AND METHODS}

This is a retrospective study of patients of alcoholic and non-alcoholic liver disease. A total of 177 patients 
(114 men and 58 women) treated as inpatients in Trichy SRM Medical College Hospital and Research Centre, were selected. About 40 patients with etiology of viral, autoimmune, and biliary lithiasis were excluded. A total of 137 patients were finalized (98 men and 39 women) and were classified into two groups, ALD (70) and NAFLD (67) based on diagnosis.

The study participants were residents of in and around Trichy, Tamil Nadu, South India. The study was conducted after the pre-approval of Institutional Ethics Committee.

The study reviewed electronic medical records of the selected patients and obtained data on AST, ALT, BMI, AGE, SEX, and MCV. Other information taken from the electronic medical records were sociodemographic factors such as gender, age, ethnicity, and other past medical, surgical, and treatment history were noted for the exclusion criteria.

\section{Exclusion criteria}

Liver disorders with viral, autoimmune, or biliary lithiasis were excluded from the study.

\section{Statistical analysis}

Data were expressed as mean \pm standard deviation. All outcomes were assessed using Chi-squared test and independent t-test, $\mathrm{P}<0.05$ was considered statistically significant. Statistical analysis were done using SPSS version 23 statistical software. Evaluation of the validity of ANI scoring system was done using area under the receiver-operating characteristic (AUROC) curve, with its $95 \%$ confidence interval. AUROC was performed assessing the sensitivity, specificity, positive predictive value, negative predictive value, and cutoff value using Med Calc v.10.20.0 (MedCalc Software, Mariakerke, Belgium). The optimal cutoff for the detection of ALD was determined according to the highest sensitivity and specificity. For all tests, significance was achieved at $\mathrm{P}$ value less than 0.05 .

The age distribution among ALD and NAFLD patients had no statistical differences $(\mathrm{P}<0.05)$. The sex distribution was different, in the ALD group,all patients being men. $(\mathrm{P}<0.01)$ ( Table 1).

In this study, BMI values were significantly lower in ALD patients in comparison with NAFLD patients $(\mathrm{P}<0.01)$.

Table 1: Sex distribution in patients with
alcoholic liver disease and non-alcoholic fatty
liver disease
\begin{tabular}{lcc}
\hline Sex & ALD (70) & NAFLD (67) \\
\hline Male & $100 \%(70 / 70)$ & $47.79 \%(28 / 67)$ \\
Female & $0 \%(0 / 70)$ & $58.20 \%(39 / 67)$ \\
\hline ALD: Alcoholic liver disease, NAFLD: Non-alcoholic fatty liver disease
\end{tabular}

The average values of biochemical parameters, $\mathrm{MCV}$, and AST/ALT ratio were significantly higher in patients with ALD compared with the patients with NAFLD $(\mathrm{P}<0.01)$. ANI was significantly higher in patients with ALD than NAFLD ( $<<0.01)$.

\section{ANI values in patients with ALD and NAFLD}

Significance of the difference was estimated using the rank-sum test $(* \mathrm{P}<0.01)$ (Figure 1$)$.

The plot of the receiver-operating characteristic (ROC) curve for ALD is presented in Figure. 2. For this ROC analysis, the AUROC showed significant discriminatory power with a $95 \%$ confidence interval $(\mathrm{P}<0.001)$.

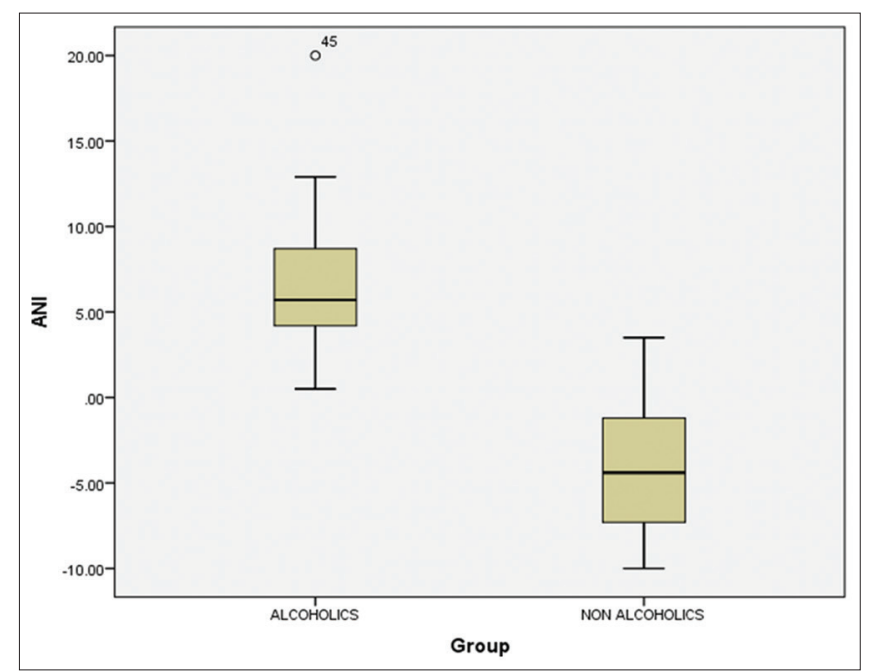

Figure 1: ANI values in patients with ALD and NAFLD

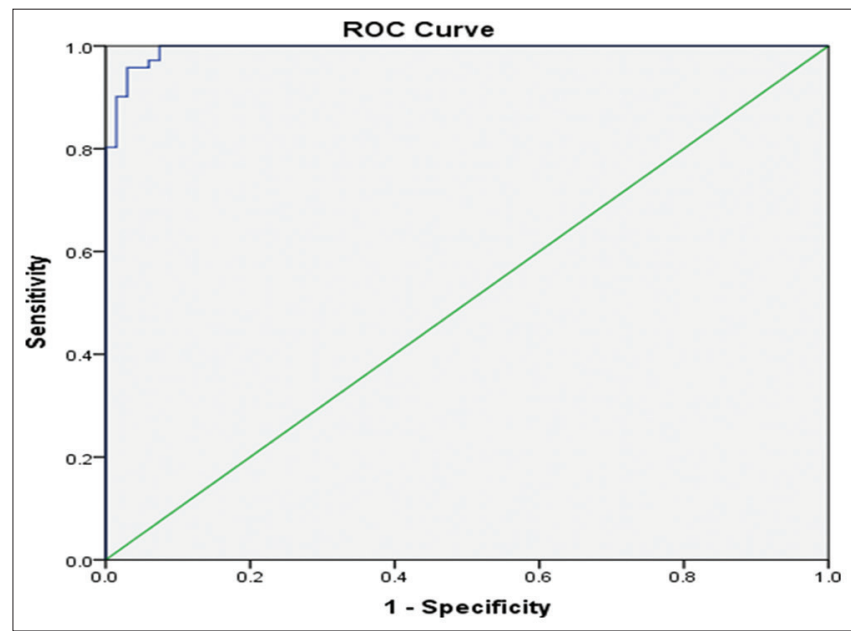

\begin{tabular}{ccclll}
\hline & ANI & AUROC & \multicolumn{2}{l}{$\begin{array}{l}\text { 95\% cofidence } \\
\text { interval }\end{array}$} & P-value \\
\hline ALD & -0.11 & 0.994 & 0.986 & 1.000 & $<0.0001$ \\
\hline
\end{tabular}

AUROC curve analysis for ANI score values in diagnosing ALD. ALD: Alcoholic liver disease, ANI: ALD/NAFLD index, AUROC: Area under receiver operating characteristic

Figure 2: Receiver-operating characteristic (ROC) curve for ALD 


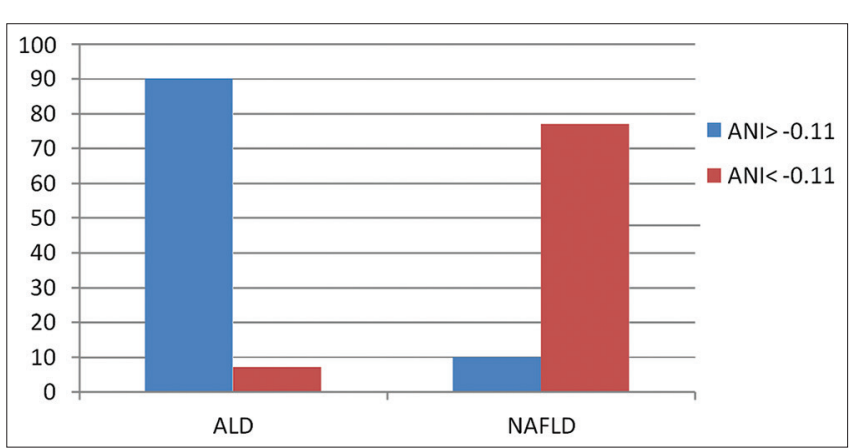

Figure 3: Distribution of ANI scores (ALD/NAFLD)

According to the ROC curve, the cut off value of ANI is - 0.11. ANI greater than -0.11 indicates ALD and ANI less than -0.11 is indicative of NAFLD, with high specificity $(91.04 \%)$ and sensitivity $(97 \%)$.

This figure shows ANI was significantly higher in patients with ALD than NAFLD $(\mathrm{P}<0.01)$.

\section{DISCUSSION}

Alcoholic liver disease (ALD) and Nonalcoholic fatty liver disease (NAFLD), though two different clinical entities, they share similar pathological spectra from simple hepatic steatosis, steatohepatitis, liver cirrhosis and liver failure. it should also be noted that both ALD and NAFLD patients have complications of cardiovascular diseases and malignancy. ${ }^{8,9}$

Though many markers such as Mast (mitochondrial aspartate transaminase), CDT (carbohydrate deficient transferrin) and Protein Kinase $\mathrm{C} \varepsilon(\mathrm{PKC}-\varepsilon)$, can also be used for the diagnosis of ALD or NAFLD, they lack sensitivity and specificity. ${ }^{10,11,12}$ But many studies have proposed, the ANI diagnostic model put forth by Dunn et al, have high accuracy in differentiating ALD and NAFLD. The advantage with this model is that,it has also taken into account, parameters of obesity,BMI. ${ }^{6,8,9}$ In this study, ALD were more common in men as alcohol abuse is more prevalent among men than in women. ${ }^{13}$ The mean BMI of patients with NAFLD was also higher than that of ALD group which is due to obesity, the major cause of NAFLD. ${ }^{14}$

The present study reveals that alcohol has a specific effect on aminotransferases. Though there is increase of ALT in other liver disorders, in Alcoholic liver disease, there is raise in AST. This is due to the fact that pyridoxal phosphate being the coenzyme for the transaminases, is mostly utilized by the ALT enzyme. Inn alcoholics, due to pyridoxine deficiency, ALT becomes much lower than AST, leading to higher AST/ALT ratio. ${ }^{15,16}$ In this study, the average MCV was greater in ALD than NAFLD, which may due to direct toxic effects of alcohol on hematopoietic cells and also due to decreased absorption of B12/ Folic acid. ${ }^{17,18}$

Our results showed that ANI was significantly higher in patients with ALD than NAFLD $(\mathrm{P}<0.01)$. which emphasize the reliability of ANI scoring system in diagnosing the types of steatohepatitis.

Eventhough, ANI scoring system, cannot replace, histopathology, it is definitely, a reliable non-invasive method for predicting ANI in a clinical set up.

Limitations of the study

Additional prospective multi-centric studies, with large sample size, along with correlation histo - pathological analysis would help in establishing the reliability of ANI scoring system.

\section{CONCLUSION}

on the basis of the results, ANI scoring system may be used in clinically distinguish ALD from NAFLD, avoiding the risks of liver biopsy. Although ANI confirms alcoholic etiology, it does not exclude other associated factors in the development of fatty liver.

\section{ACKNOWLEDGMENT}

We would like to thank Medical Records Department of Trichy SRM Medical College Hospital and Research Centre, Trichy for their assistance in this study.

\section{REFERENCES}

1. Day CP. Genes or environment to determine alcoholic liver disease and non-alcoholic fatty liver disease. Liver Int. 2006;26(9):1021-1028.

http://doi.org/10.1111/j.1478-3231.2006.01323.x

2. Clark JM. The epidemiology of nonalcoholic fatty liver disease in adults. J Clin Gastroenterol. 2006;40(Suppl 1):S5-S10. http://doi.org/10.1097/01.mcg.0000168638.84840.ff

3. Targher G, Bertolini L, Padovani R, Rodella S, Tessari R, Zenari L, Day $\mathrm{C}$, et al. fatty liver disease and its association withcardiovascular diseaseamong type 2 diabetic patients. Diabetes Care. 2007;30(5):1212-1218.

http://doi.org/10.2337/dc06-2247

4. Frazier TH, Stocker AM, Kershner NA, Marsano LS and McClain CJ. Treatment of alcoholic liver disease. Therap Adv Gastroenterol. 2011;4(1):63-81. http://doi.org/10.1177/1756283X10378925

5. Brunt EM and Tiniakos DG. Histopathology of nonalcoholic fatty liver disease. World J Gastroenterol. 2010;16(42):5286-5296. http://doi.org/10.3748/wjg.v16.i42.5286

6. Pulzi FB, Cisternas R, Melo MR, Ribeiro CM, Malheiros CA 
and Salles JE. New clinical score to diagnose nonalcoholic steatohepatitis in obese patients. Diabetol Metab Syndr. 2011;3(1):3.

http://doi.org/10.1186/1758-5996-3-3

7. Dunn W, Angulo P, Sanderson S, Jamil LH, Stadheim L, Rosen C, et al. Utility of a new model to diagnose an alcohol basis of steatohepatitis. Gastroenterology 2006;131(4):10571063.

http://doi.org/10.1053/j.gastro.2006.08.020

8. Adams LA, Lymp JE, Sauver JS, Sanderson SO, Lindor KD, Feldstein $A$, et al. The natural history of nonalcoholic fatty liver disease: A population-based cohort study. Gastroenterology. 2005;129(1):113-121.

http://doi.org/10.1053/j.gastro.2005.04.014

9. Cerović I, Mladenović D, Ješić R, Naumović T, Branković M, Vučević D, et al. Alcoholic liver disease/nonalcoholic fatty liver disease index: Distinguishing alcoholic from nonalcoholic fatty liver disease. Eur J Gastroenterol Hepatol. 2013;25(8):899-904. http://doi.org/10.1097/MEG.0b013e32835f0786

10. Macchia T, Mancinelli R, Gentili S, Ceccanti M, Devito R, Attilia ML, et al. Mitochondrial aspartate aminotransferase isoenzyme: A biochemical marker for the clinical management of alcoholics? Clin Chim Acta. 1997;263(1):79-96. http://doi.org/10.1016/s0009-8981(97)00046-6

11. Imbert-Bismut $F$, Naveau S, Morra R, Munteanu M, Ratziu V, Abella A, et al. The diagnostic value of combining carbohydratedeficient transferrin, fibrosis, and steatosis biomarkers for the prediction of excessive alcohol consumption. Eur J Gastroenterol Hepatol. 2009;21(1):18-27.

http://doi.org/10.1097/MEG.0b013e32830a4f4c
12. Kaiser JP, Beier JI, Zhang J, Hoetker JD, von Montfort C, Guo L, et al. PKCepsilon plays a causal role in acute ethanol-induced steatosis. Arch Biochem Biophys. 2009;482(1-2):104-111. http://doi.org/10.1016/j.abb.2008.11.004

13. Mandayam S, Jamal MM and Morgan TR. Epidemiology of alcoholic liver disease. Semin Liver Dis. 2004;24(3):217-232. http://doi.org/10.1055/s-2004-832936

14. Preiss D and Sattar N. Non-alcoholic fatty liver disease: an overview of prevalence, diagnosis, pathogenesis and treatment considerations. Clin Sci (Lond). 2008;115(5):141-150. http://doi.org/10.1042/CS20070402

15. Tutor-Crespo MJ, Hermida $\mathrm{J}$ and Tutor JC. Activation of serum aminotransferases by pyridoxal-5' -phosphate in epileptic patients treated with anticonvulsant drugs. Clin Biochem. 2004;37(8):714-717. http://doi.org/10.1016/j.clinbiochem.2004.03.007

16. Vermaak WJ, Barnard HC, van Dalen EM and Potgieter GM. Correlation between pyridoxal-5'-phosphate levels and the percentage activation of aspartate aminotransferase enzyme in haemolysate and plasma during in vitro incubation studies with different B6 vitamers. Enzyme. 1986;35(4):215-224. http://doi.org/10.1159/000469345

17. Maruyama S, Hirayama C, Yamamoto S, Koda M, Udagawa A, et al. Red blood cell status in alcoholic and non-alcoholic liver disease. J Lab Clin Med. 2001;138(5):332-337. http://doi.org/10.1067/mlc.2001.119106

18. Cravo ML and Camilo ME. Hyperhomocysteinemia in chronic alcoholism: Relations to folic acid and Vitamins $B(6)$ and $B(12)$ status. Nutrition. 2000;16(4):296-302. http://doi.org/10.1016/s0899-9007(99)00297-x

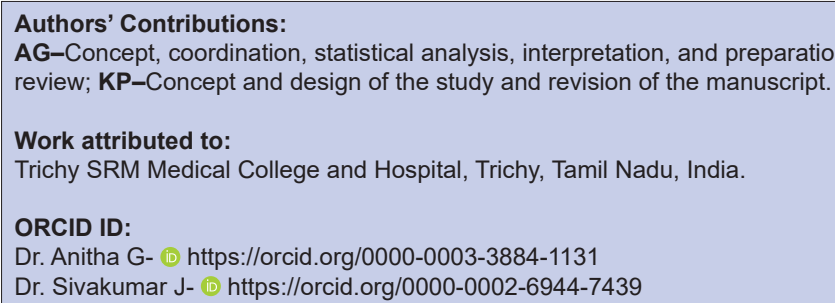

